\title{
COMMUTING TRANSFORMATIONS AND MIXING ${ }^{1}$
}

\section{A. AKCOGLU, R. v. CHACON AND T. SCHWARTZBAUER}

1. Introduction. The main purpose of this paper is to prove the following theorem: If two invertible measure preserving transformations commute, and the first admits an approximation by partitions, then the second is the limit of sums, composed of at most two terms, of positive and negative powers of the first.

The main theorem of the paper is a generalization of a result given in [1] where it is indicated that the result there can be applied to yield a simplification of the construction given in [2] of a transformation having continuous spectrum and no roots.

As an application, we show that the main result yields a way of constructing a transformation which is strongly mixing, and which therefore has continuous spectrum, and which commutes only with its powers (having, therefore, no roots). The striking result that such transformations exist was first obtained by Ornstein in [3].

2. Definitions and preliminaries. Let $(X, F, \mu)$ be a normalized nonatomic Lebesgue space (i.e., a space isomorphic to the unit interval). As usual, all sets that are referred to are understood to be in $\mathcal{F}$, even if this is not explicitly stated.

Definition 2.1. A collection $\xi$ of sets having union $X_{\xi} \subset X$ will be called a partition if the sets are in $\mathcal{F}$ and are pairwise disjoint. $\mathcal{F}(\xi)$ will denote the $\sigma$-field of subsets of $X_{\xi}$ generated by $\xi$. If $\xi=\left\{A_{0}, A_{1}, A_{2}, \cdots\right\}$ is a (finite or) countable partition, then any set $A \in \mathcal{F}(\xi)$ can be written as $A=\sum_{i=0}^{\infty} a_{i} A_{i}$ where $a_{i}, i=0,1, \cdots$, is equal to zero or one, with the convention that $1 B=B, 0 B=\varnothing$ for any set $B$.

Definition 2.2. If $\xi$ is a countable partition and $A \in \mathcal{F}$, then among the sets in $\mathcal{F}(\xi)$ there is at least one whose symmetric difference with $A$ has minimal measure. We denote by $A(\xi)$ any one of these sets.

Definition 2.3. If $F, F_{n} \in \mathcal{F}$, we write $\lim _{n \rightarrow \infty} F_{n}=F$ provided that $\lim _{n \rightarrow \infty} \mu\left(F \Delta F_{n}\right)=0$. If $\xi_{n}$ is a sequence of countable partitions, we write $\xi_{n} \rightarrow \epsilon$ if for any $A \in \mathcal{F}, \lim _{n \rightarrow \infty} A\left(\xi_{n}\right)=A$.

Definition 2.4. Given an invertible and measure preserving transformation $\tau$ of $X$, we say that a partition $\xi$ is $\tau$-admissible if $\xi=\left\{A_{0}, A_{1}, \cdots, A_{q}\right\}$ is finite and $\tau A_{i-1}=A_{i}, 1 \leqq i \leqq q$. The trans-

Received by the editors April 16, 1969.

1 Research supported in part by NSF grant GP-3752 and by NRC grant A3974. 
formation $\tau$ is said to have an approximation by partitions if there is a sequence $\xi_{n}=\left\{A_{i}(n): i=0,1, \cdots, q(n)\right\}$ of $\tau$-admissible partitions such that $\xi_{n} \rightarrow \epsilon$. If, in addition, $\lim _{n \rightarrow \infty} \mu\left(\tau A_{q(n)}(n) \Delta A_{0}(n)\right) / \mu\left(A_{0}(n)\right)$ $=0$, then we say that $\tau$ has a strong approximation by partitions.

3. Main result. In what follows, $\tau$ and $\sigma$ are two commuting invertible measure preserving transformations of $X$. For $A, B \in \mathcal{F}$, we write $A=B+E(\epsilon)$ provided that $\mu(A \Delta B) \leqq \epsilon$.

Lemma 3.1. Let $\xi=\left\{A_{0}, A_{1}, \cdots, A_{q}\right\}$ be $\tau$-admissible, $A, B \in \mathcal{F}(\xi)$ and $\sigma A=B+E(\epsilon)$. Then there are two disjoint sets $F$ and $G$ and two nonnegative integers $j, k$, such that $\sigma A=\tau^{j}(A \cap F)+\tau^{-k}(A \cap G)+E(2 \epsilon)$, $j+k \geqq q+1$ and $\mu(F) \leqq k / q+1, \mu(G) \leqq j / q+1$.

Proof. Let $C_{i}=A_{0} \cap \sigma \tau^{i} A_{0},-q \leqq i \leqq q$. If $x \in C_{i} \cap C_{j}$, then there exist two points $y, z \in A_{0}$ such that $x=\sigma \tau^{i} y=\sigma \tau^{j} z$, which implies that $\tau^{i-j} y=z$. Hence either $i=j$ or $|i-j| \geqq q+1$. Therefore any $q+1$ consecutive $C_{i}$ 's are pairwise disjoint.

Now, if we let $R=X-X_{\xi}$, then for any $l, 0 \leqq l \leqq q$,

$$
\sigma A_{l}=\sum_{k=0}^{q} A_{k} \cap \sigma A_{l}+R \cap \sigma A_{l}=\sum_{k=0}^{q} \tau^{k} C_{l-k}+R \cap \sigma A_{l} .
$$

Hence, by writing

$$
A=\sum_{l=0}^{q} a_{l} A_{l}, \quad B=\sum_{k=0}^{q} b_{k} A_{k}=\sum_{k=0}^{q} \tau^{k} b_{k} A_{0},
$$

we obtain

$$
\sigma A=\sum_{k=0}^{q} \tau^{k} \sum_{l=0}^{q} a_{l} C_{l-k}+R \cap \sigma A=\sum_{k=0}^{q} \tau^{k} b_{k} A_{0}+E(\epsilon),
$$

or

$$
\sum_{k=0}^{q} \tau^{k} \sum_{l=0}^{q} a_{l} C_{l-k}=\sum_{k=0}^{q} \tau^{k} b_{k} A_{0}+E(\epsilon) .
$$

Since for a fixed $k$, the sets $C_{l-k}, 0 \leqq l \leqq q$, are pairwise disjoint, this means that

$$
\sum_{k=0}^{q} \int_{A_{0}}\left|\sum_{l=0}^{q} a_{l} \Psi_{l-k}-b_{k}\right| d \mu<\epsilon
$$

where $\Psi_{i}$ is the characteristic function of $C_{i}$.

Therefore, there exists a point $x_{0} \in A_{0}$ such that 


$$
\sum_{k=0}^{q}\left|\sum_{l=0}^{q} a_{l} \Psi_{l-k}\left(x_{0}\right)-b_{k}\right| \mu\left(A_{0}\right)<\epsilon,
$$

which means that $D=B+E(\epsilon)$ where

$$
D=\sum_{k=0}^{q} \sum_{l=0}^{q} a_{l} \Psi_{l-k}\left(x_{0}\right) A_{k} .
$$

Hence we have that $\sigma A=D+E(2 \epsilon)$.

To see that this implies the lemma, we rearrange the double summation for $D$ as follows:

$$
D=\Psi_{0}\left(x_{0}\right) A+\sum_{j=1}^{q} \Psi_{-j}\left(x_{0}\right) \tau^{j} \sum_{l=0}^{q-j} a_{l} A_{l}+\sum_{k=1}^{q} \Psi_{k}\left(x_{0}\right) \tau^{-k} \sum_{l=k}^{q} a_{l} A_{l},
$$

where there are at most two nonzero terms. If there are less than two nonzero terms, we may define $F=\varnothing$ or $G=\varnothing$ or $F=G=\varnothing$ and see that all the conclusions of the lemma are satisfied. If there are exactly two nonzero terms, then

$$
D=\tau^{j} \sum_{l=0}^{q-j} a_{l} A_{l}+\tau^{-k} \sum_{l=k}^{q} a_{l} A_{l},
$$

where $j, k \geqq 1$ and $j+k \geqq q+1$ since $C_{-j} \cap C_{k} \neq \varnothing$ implies $j+k \geqq q+1$ because any $q+1$ consecutive $C_{i}$ 's are pairwise disjoint. In this case we let $F=\sum_{l=0}^{q-j} A_{l}, G=\sum_{l=k}^{q} A_{l}$, and we have

$$
\mu(F) \leqq \frac{q-j+1}{q+1} \mu\left(X_{\xi}\right) \leqq \frac{k}{q+1},
$$

and similarly $\mu(G) \leqq j / q+1$.

We may now state and prove

THEOREM 3.1. If $\tau$ has an approximation by partitions and $\sigma$ commutes with $\tau$, then there are two sequences $X_{n}^{1}, X_{n}^{2}$ of sets and two sequences of nonnegative integers $j_{n}^{1}, j_{n}^{2}, n=1,2, \cdots$, such that $X_{n}^{1} \cap X_{n}^{2}=\varnothing$ and such that:

(i) if $A \in \mathcal{F}$, then

$$
\sigma A=\lim _{n \rightarrow \infty}\left\{\tau^{j_{n}^{1}}\left(A \cap X_{n}^{1}\right)+\tau^{-j_{n}^{2}}\left(A \cap X_{n}^{2}\right)\right\}
$$

and

(ii) either $\sigma=\tau^{k}$ for some integer $k$, or $\lim _{n \rightarrow \infty} j_{n}^{1}=\lim _{n \rightarrow \infty} j_{n}^{2}=\infty$.

Proof. Let $\xi_{n}=\left\{A_{0}(n), A_{1}(n), \cdots, A_{q(n)}(n)\right\}, \xi_{n} \rightarrow \epsilon$, be a sequence of $\tau$-admissible partitions. Note that $\lim _{n \rightarrow \infty} q(n)=\infty$. Choose a se- 
quence $\epsilon_{n}>0$ such that $\epsilon_{n} q(n) \rightarrow 0$ as $n \rightarrow \infty$. For any integer $n=1,2, \cdots$, we can find another integer $m(n)$ with the following properties:

(a) there exist $B_{n}, D_{n} \in \mathcal{F}\left(\xi_{m(n)}\right)$ such that $A_{0}(n)=B_{n}+E\left(\epsilon_{n}\right)$, $\sigma A_{0}(n)=D_{n}+E\left(\epsilon_{n}\right)$, and

(b) $q(n) \leqq \epsilon_{n} q(m(n))$.

We then have that $\sigma B_{n}=D_{n}+E\left(2 \epsilon_{n}\right)$. Now choose $j_{n}^{1}$ and $j_{n}^{2}$ and $F_{n}$, $G_{n}$ according to Lemma 3.1, with respect to the partition $\xi_{m(n)}$ and the set $B_{n} \in \mathcal{F}\left(\xi_{m(n)}\right)$. Hence, $\sigma B_{n}=\tau_{n}^{j_{n}^{1}}\left(B_{n} \cap F_{n}\right)+\tau^{-j_{n}^{2}}\left(B_{n} \cap G_{n}\right)+E\left(4 \epsilon_{n}\right)$, and therefore

$$
\sigma A_{0}(n)=\tau^{j_{n}^{1}}\left(A_{0}(n) \cap F_{n}\right)+\tau^{-j_{n}^{2}}\left(A_{0}(n) \cap G_{n}\right)+E\left(7 \epsilon_{n}\right) .
$$

If we now let $X_{n}^{1}=\sum_{k=0}^{q(n)} \tau^{k}\left(A_{0}(n) \cap F_{n}\right)$ and $X_{n}^{2}=\sum_{k=0}^{q(n)} \tau^{k}\left(A_{0}(n) \cap G_{n}\right)$, we have that, for any $A \in \mathcal{F}\left(\xi_{n}\right)$,

$$
\sigma A=\tau^{j_{n}^{1}}\left(A \cap X_{n}^{1}\right)+\tau^{-j_{n}^{2}}\left(A \cap X_{n}^{2}\right)+E\left(7(q(n)+1) \epsilon_{n}\right),
$$

and it is clear that this implies (i).

To prove (ii), note that, for example

$$
\mu\left(X_{n}^{2}\right) \leqq(q(n)+1) \mu\left(G_{n}\right) \leqq(q(n)+1 / q(n(m))+1) j_{n}^{1},
$$

which means that $\lim \inf _{n \rightarrow \infty} \mu\left(X_{n}^{2}\right) \leqq \lim \inf _{n \rightarrow \infty}\left(\epsilon_{n} j_{n}^{1}\right)$. Therefore if $j_{n}^{1}$ has a bounded subsequence, considering a subsequence if necessary, one may assume that $X_{n}^{2}=\varnothing$ and $j_{n}^{1}=k$ for all $n$.

As an application of this theorem, we give the following result.

THEOREM 3.2. If a mixing transformation $\tau$ has an approximation by partitions, then it can only commute with its powers.

Before the proof, we note that the nontrivial fact that there are mixing transformations that have an approximation by partitions follows from the results given in [3]. Hence Theorem 3.2 is not vacuously true.

Proof. Using the notation of Theorem 3.1, we have $\sigma A \subset \tau^{j_{n}^{1}} A$ $+\tau^{-i_{n}^{2} A}+E_{n}$ for all $n \geqq 1$ where $\mu\left(E_{n}\right) \rightarrow 0$. Hence

$$
\sigma A \subset\left\{\tau^{j_{n}^{1}} A+\tau^{-j_{n}^{2}} A+E_{n}\right\} \cap\left\{\tau^{j_{m}^{1}} A+\tau^{-j_{m}^{2}} A+E_{m}\right\},
$$

or

$$
\begin{gathered}
\sigma A \subset \tau^{j_{n}^{1}} A \cap \tau^{j_{m}^{1}} A+\tau^{j_{n}^{1}} A \cap \tau^{-j_{m}^{2}} A+\tau^{-j_{n}^{2}} A \cap \tau^{j_{m}^{1}} A \\
+\tau^{-j_{n}^{2}} A \cap \tau^{-j_{m}^{2}} A+E_{m n}
\end{gathered}
$$


where $\mu\left(E_{m n}\right) \rightarrow 0$ as $m, n \rightarrow \infty$. Now, if $\sigma$ is not a power of $\tau$, then $j_{n}^{1}, j_{n}^{2} \rightarrow \infty$ as $n \rightarrow \infty$. Hence, letting first $n \rightarrow \infty$ then $m \rightarrow \infty$, we see, from the mixing property,

$$
\mu(\sigma A)=\mu(A) \leqq 4[\mu(A)]^{2} \quad \text { for all } A \in \mathcal{F},
$$

which is a contradiction.

Lemma 3.2. If, in addition to the hypotheses of Lemma 3.1, $A_{0}=\tau A_{a}$ $+E(\delta)$, then there exists an integer $j,-q \leqq j \leqq q$ and $a$ set $A^{\prime} \subset A$ such that $\sigma A=\tau^{i} A^{\prime}+E(2 \epsilon+2(q+1) \delta)$.

Proof. We already have that $\sigma A=D+E(2 \epsilon)$. If $D=\varnothing$ or $D$ contains only one nonzero term, the lemma follows. Also, if $D=\tau^{j} \sum_{l=0}^{q-j} a_{l} A_{l}+\tau^{-k} \sum_{l=k}^{q} a_{l} A_{l}$ where $j+k=q+1$, we may write $\tau^{-k} A_{l}$ $=\tau^{j} \tau^{-q-1} A_{l}=\tau^{i} A_{l}+E(\delta)$, which means that $D=\tau^{i} A+E((q+1) \delta)$, and again the lemma follows. Therefore, the only case which need be considered is the case for which $\Psi_{-j}\left(x_{0}\right)+\Psi_{k}\left(x_{0}\right)=2$ for some $j, k \geqq 1$ with $j+k>q+1$.

We will now give an upper bound for the measure of the set

$$
S=\left\{x \mid \exists j, k, 1 \leqq j, k \leqq q, 1+q<j+k, \Psi_{-j}(x)+\Psi_{k}(x)=2\right\} .
$$

By the definition of $S$,

$$
\begin{aligned}
S & =\sum_{j=2}^{q} \sum_{k=q-j+2}^{q} C_{-j} \cap C_{k}=\sum_{j=2}^{q} \sum_{k=q-j+2}^{q} A_{0} \cap \tau^{-j} \sigma A_{0} \cap \tau^{k} \sigma A_{0} \\
& =\sum_{j=2}^{q} \tau^{-j}\left\{A_{j} \cap \sigma\left(A_{0} \cap \sum_{k=q-j+2}^{q} \tau^{k+j} A_{0}\right)\right\} \\
& =\sum_{j=2}^{q} \tau^{-j}\left\{A_{j} \cap \sigma\left(A_{0} \cap \sum_{l=2}^{j} \tau^{l} A_{q}\right)\right\}
\end{aligned}
$$

which means that $\mu(S) \leqq \mu\left(A_{0} \cap \sum_{l=1}^{q-1} \tau^{1+l} A_{q}\right)$. Hence $\mu\left(A_{0} \cap \tau A_{q}\right)$ $>\mu\left(A_{0}\right)-\delta$ implies that $\mu(S)<\delta$.

Now going back to

$$
\int_{A_{0}} \sum_{k=0}^{q}\left|\sum_{l=0}^{q} a_{l} \Psi_{l-k}-b_{k}\right| d \mu<\epsilon,
$$

we see that there exists a point $x_{0} \in A_{0}-S$ such that

$$
\sum_{k=0}^{q}\left|\sum_{l=0}^{q} a_{l} \Psi_{l-k}\left(x_{0}\right)-b_{k}\right| \mu\left(A_{0}\right)<\epsilon+(q+1) \delta,
$$

since the integrand is bounded by $q+1$. This completes the proof. 
The next theorem is proved in [1], but we include a simpler proof for the sake of completeness.

THEOREM 3.3. If $\tau$ has a strong approximation by partitions and $\sigma$ commutes with $\sigma$, then there is a sequence of nonnegative integers $j_{n}$, $n=1,2, \cdots$, such that if $A \in \mathcal{F}$ then

$$
\sigma(A)=\lim _{n \rightarrow \infty} \tau^{j n}(A) .
$$

Proof. The proof is the same as the proof of Theorem 3.1 with Lemma 3.2 replacing Lemma 3.1.

\section{REFERENCES}

1. R. V. Chacon and T. Schwartzbauer, Commuting point transformations, Z. Wahrscheinlichkeitstheorie und Verw. Gebeite 11 (1969), 277-287.

2. R. V. Chacon, A geometric construction of measure preserving transformations, Proc. Fifth Berkeley Sympos. Math. Statis. and Prob. (Berkeley, Calif., 1965/66) vol. II : Contributions to Prob. Theory, part 2, Univ. of California Press, Berkeley, Calif., 1967, pp. 335-360. MR 35 \#3033.

3. D. Ornstein, A mixing transformation that commutes only with its powers (to appear).

UNIVERSITY OF MINNESOTA AND

UNIVERSITY OF TORONTO 